\title{
O desenvolvimento de doenças psiquiátricas em crianças após o diagnóstico de câncer: uma revisão integrativa
}

\author{
The emergence of psychiatric illnesses in children after cancer diagnosis: a integrative review \\ Aparición de psicopatologias em niños después del diagnóstico de cancer: una revision \\ integradora
}

Anna Lillian Canuto Bittencourt ${ }^{1 *}$, Ana Cláudia Leal Cavalcanti ${ }^{2}$, Andreane Meneses Andrade ${ }^{1}$, Anne Caroline Araújo Almeida ${ }^{1}$, Beatriz Luduvice Soares ${ }^{1}$, Bruna Aparecida Siqueira da Silva ${ }^{3}$, Fernanda Fontes Prado Reis ${ }^{2}$, Letycia Santos Rodrigues ${ }^{1}$, Pedro Reges Pereira Meira ${ }^{2}$, lane Brito Leal ${ }^{1}$.

\section{RESUMO}

Objetivo: Realizar um levantamento bibliográfico sobre o desenvolvimento de doenças psiquiátricas em crianças após o diagnóstico de câncer. Métodos: Trata-se de uma revisão integrativa da literatura realizada na base de dados em saúde PubMed. Estratégias de busca foram realizadas com a utilização dos seguintes descritores em inglês: "Cancer", "Child", "Mental Health" AND "Psychopathology. Resultados: A primeira estratégia de busca com os descritores "Cancer", "Child" AND "Mental Health", foram encontrados 2.204 estudos científicos. Destes, foram selecionadas 2.051 produções que apresentavam o texto na íntegra, 1.646 relativos ao ano de publicação (entre 2013 e 2020) e 1.314 pertinente a espécie humana. Após a seleção por triagem pelos títulos e resumos restaram apenas 6 artigos científicos. A segunda estratégia de busca utilizou os descritores "Cancer", "Child" AND "Psychopathology" e foram encontrados 130 artigos com os mesmos critérios de busca citados anteriormente. Desses, foram selecionadas 4 produções. Considerações finais: $O$ biopsicossocial da criança, que ainda se encontra em fase de adaptação após o diagnóstico da doença, sofre uma mudança brusca da rotina, sendo, por isso, os altos índices de transtornos de ansiedade e de depressão. Assim, elaborar e difundir o conhecimento sobre as psicopatologias nas crianças diagnosticadas com câncer é de suma importância.

Palavras-chave: Câncer, Criança, Saúde mental, Psicopatologia.

\section{ABSTRACT}

Objective: carry out a bibliographic survey on the development of psychiatric diseases in children after the diagnosis of cancer. Methods: This is an integrative literature review carried out in the PubMed health database. Search strategies were carried out using the following descriptors in English: "Cancer", "Child", "Mental Health" AND "Psychopathology. Results: The first search strategy with the keywords "Cancer", "Child" AND "Mental Health", 2,204 scientific studies were found. Of these, 2,051 productions were selected that presented the full text, 1,646 for the year of publication (between 2013 and 2020) and 1,314 for the human species. After the selection by sorting by titles and abstracts, only 6 scientific articles remained. The second search strategy uses the descriptors "Cancer", "Child" AND "Psychopathology" and 130 articles were found with the same search criteria mentioned above. Of these, 4 productions were selected. Final considerations: The child's biopsychosocial, who is still in the adaptation phase after the diagnosis of the disease, undergoes a sudden change in routine, which is why the high rates of anxiety and depression disorders. Thus, developing and disseminating knowledge about psychopathologies in children diagnosed with cancer is of paramount importance.

Keywords: Cancer, Child, Mental health, Psychopathology.

\section{RESUMEN}

Objetivo: Realizar una encuesta bibliográfica sobre el desarrollo de enfermedades psiquiátricas en niños tras el diagnóstico de cáncer. Métodos: Se trata de una revisión integrativa de la literatura realizada en la base de datos de salud PubMed. Las estrategias de búsqueda se llevaron a cabo utilizando los siguientes descriptores en inglés: "Cancer", "Child", "Mental Health" Y "Psychopathology". Resultados: Se encontró la primera estrategia de búsqueda con las palabras clave "Cáncer", "Niño" Y "Salud Mental", se encontraron 2.204 estudios científicos. De estas, se seleccionaron 2.051 producciones que presentaron el texto completo, 1.646 para el año de publicación (entre 2013 y 2020 ) y 1.314 para la especie humana. Después de la selección ordenada por títulos y resúmenes, solo quedaron 6 artículos científicos. La segunda estrategia de búsqueda utiliza los descriptores "Cancer", "Child" AND "Psychopathology" y se encontraron 130 artículos con los mismos criterios de búsqueda mencionados anteriormente. De estas, se seleccionaron 4 producciones. Consideraciones finales: El niño biopsicosocial, que aún se encuentra en fase de adaptación tras el diagnóstico de la enfermedad, sufre un cambio brusco de rutina, de ahí las altas tasas de trastornos de ansiedad y depresión. Por tanto, desarrollar y difundir conocimientos sobre psicopatologías en niños diagnosticados de cáncer es de suma importancia.

Palabras clave: Cáncer, Niño, Salud mental, Psicopatología.

${ }^{1}$ Universidade Federal de Sergipe (UFS), Lagarto - SE. *E-mail: annalilliancanuto@gmail.com

2Universidade Tiradentes (UNIT), Aracaju - SE.

${ }^{3}$ União Metropolitana de Educação e Cultura (UNIME), Lauro de Freitas - BA.

${ }^{4}$ Universidade Federal de Sergipe (UFS), São Cristóvão - SE. 


\section{INTRODUÇÃO}

O câncer é uma das principais causas de mortalidade infantil no mundo, com cerca de 300.000 novos casos por ano, e é responsável por causar uma grande limitação nos pacientes, pela própria doença e pelo seu tratamento. A criança tem a sua fisiologia do organismo alterada e, ainda, pode desenvolver algumas psicopatologias, já que possui uma alteração na sua rotina e em seus hábitos comuns da própria infância (ANDERS JC e SOUZA AIJ, 2009; BELL H, et al., 2018; MAVRIDES N e PAO M, 2014; WIENER L, et al., 2014; WHO,2018).

A infância é um período de grande importante na vida de um indivíduo. É nela que, a partir de todas as vivências com o meio externo, a criança adquire a sua personalidade, a qual vai ser base de todas as suas experiências futuras. Apresentar uma doença, em qualquer idade, é indesejável e gera medo, principalmente o câncer, dependendo do tipo e do diagnóstico precoce (CARDOSO FT, 2007; CASTRO EH, 2010).

$\mathrm{O}$ tratamento contra o câncer possui algumas particularidades que podem levar ao desenvolvimento de psicopatologias. Um exemplo disso é o desenvolvimento de um transtorno de ansiedade devido ao distanciamento da criança de sua casa, por hospitalização, e da escola, e, ainda, ao seu caráter doloroso que pode gerar um sentimento de impotência, medo, fobia e estresse na criança acometida (BELL H, et al., 2018; CICOGNA EC, et al., 2010; MAVRIDES N e PAO M, 2014).

Dessa forma, a solidão, o isolamento, a perda de uma infância normal, o desconforto físico, a incapacidade de realizar algumas atividades sozinhos e, por fim, respostas emocionais, como a raiva e o medo, são exemplos de comportamentos que a criança passa a ter ao receber o diagnóstico de câncer. Ela e seus familiares compartilham todos esses sentimentos e as suas vidas são transformadas após a descoberta (BELL H, et al., 2018; CICOGNA EC, et al., 2010; MAVRIDES N e PAO M, 2014; WIENER L, et al., 2014).

É importante ressaltar que a associação entre uma psicopatologia e o câncer pode causar uma piora na evolução da doença de base, pois pode levar a uma menor aderência às terapias, aumento de dores inexplicáveis, além de uma maior morbimortalidade. Deve haver uma atenção maior, no caso da depressão, porque sintomas depressivos também podem ocorrer em doenças crônicas, fazendo com que ela seja subdiagnosticada e subtratada. É necessária uma avaliação adequada desses sintomas, que podem estar superpostos aos sintomas da patologia clínica (CARDOSO FT, 2007; CICOGNA EC, et al., 2010; OLIVEIRA J, 2009).

Assim, é de grande importância que os profissionais de saúde procurem entender todos os aspectos que envolvem essa enfermidade, além do biológico, para que a sua relação com o paciente e sua família seja a mais completa e, principalmente, a mais humana possível (CARDOSO FT, 2007; CICOGNA EC, et al., 2010).

Com base no exposto acima, é essencial o conhecimento das psicopatologias desenvolvidas por uma criança após o seu diagnóstico de câncer. Sendo assim, o objetivo desse trabalho foi realizar um levantamento bibliográfico sobre o desenvolvimento de doenças psiquiátricas em crianças após o diagnóstico de câncer.

\section{MÉTODOS}

Trata-se de uma revisão integrativa da literatura em que a identificação e seleção dos estudos ocorreram entre os meses agosto de 2018 e julho de 2020.

Foi realizada uma busca na base de dados em saúde: PubMed. A estratégia de busca foi realizada com os seguintes descritores em inglês, "Cancer", "Child", "Mental Health" AND "Psychopathology".

Foram considerados elegíveis estudos publicados em inglês, português e espanhol, nos últimos dez anos e disponível em formato eletrônico. Trabalhos duplicados na base de dados e que não tratem sobre Psicopatologias e Câncer em crianças em seus objetivos foram excluídos (Figura 1).

O processo de revisão consistiu em dois níveis de triagem, a revisão de título e resumo e a revisão do texto na íntegra. Para o primeiro nível, os títulos e resumos foram lidos e analisados para identificar artigos potencialmente elegíveis. Na segunda etapa, os artigos foram lidos na íntegra para determinar se atendem aos critérios de elegibilidade. 
Todas as referências foram revisadas por completo e extraído fragmentos relevantes para o trabalho em forma de citação. Desta maneira, não houve necessidade de aprovação pelo Comitê de Ética em Pesquisa (CEP), por não se tratar de estudo experimental em seres humanos.

Figura 1 - Critérios de inclusão e exclusão.

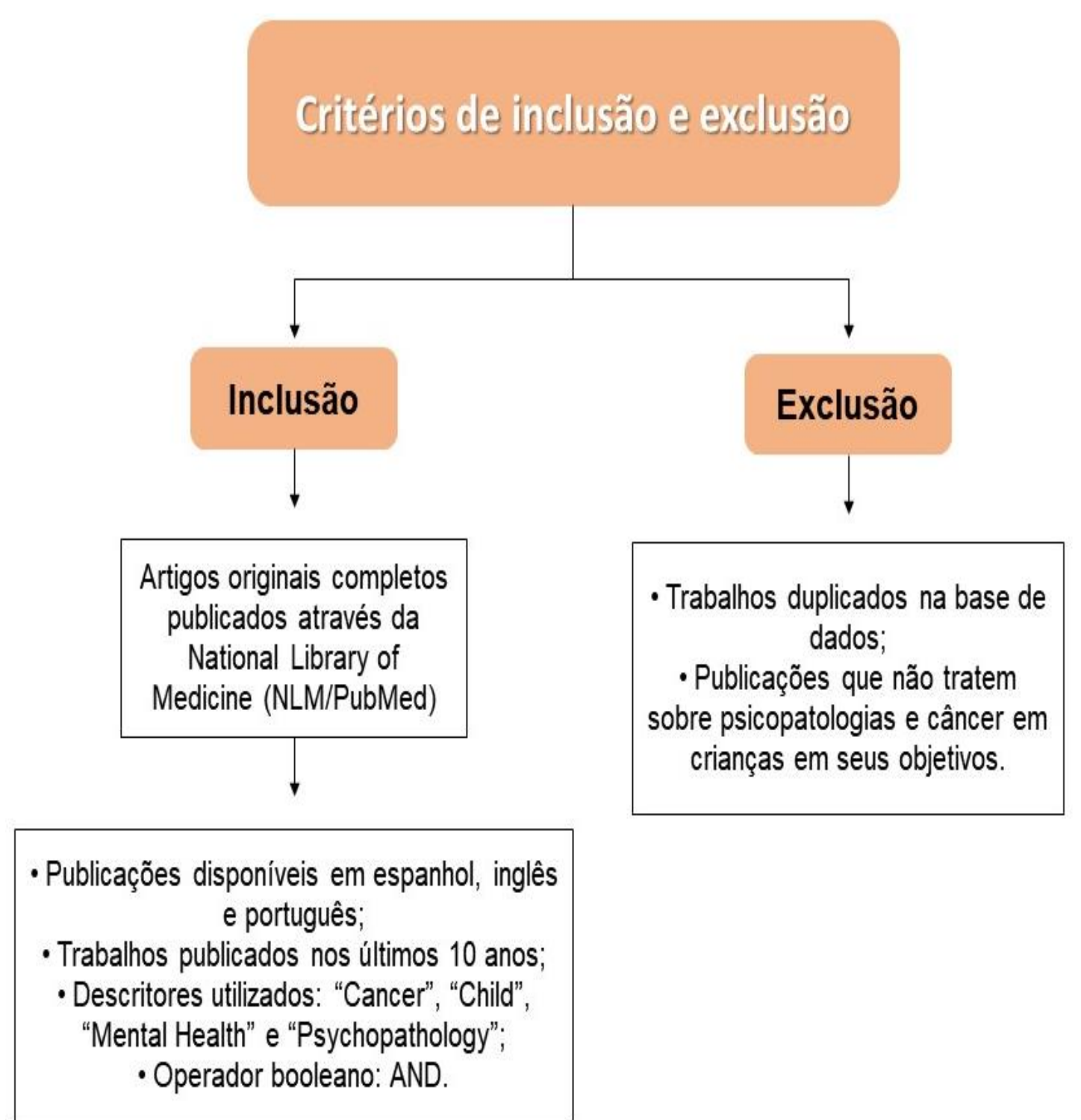

Fonte: Bittencourt ALC, et al., 2020.

\section{RESULTADOS E DISCUSSÃO}

Inicialmente foram encontradas 2.204 produções científicas com os descritores "Cancer", "Child" AND "Mental Health". Destas, foram selecionadas 2.051 produções que apresentavam o texto na íntegra, disponível online. Apenas 1.646 atenderam aos critérios de elegibilidade relativo ao ano da publicação. Ao limitar a busca para espécie humana, restaram 1.314 artigos. Destes, 6 atenderam aos critérios de elegibilidade.

Ao utilizar os descritores "Cancer", "Child" AND "Psychopathology" foram encontrados 130 estudos científicos que, após aplicação dos critérios supracitados, foram selecionadas 4 produções.

Para extração dos dados dos artigos selecionados foi elaborada uma ficha padrão com campos para coleta dos dados como, título, autores, ano de publicação, revista e tipo de estudo (Quadro 1). 
Quadro 1 - Estudos incluídos na revisão bibliográfica e suas principais características.

\begin{tabular}{|c|c|c|c|c|}
\hline TÍTULO & AUTORES & $\begin{array}{c}\text { ANO DE } \\
\text { PUBLICAÇÃO }\end{array}$ & REVISTA & TIPO DE ESTUDO \\
\hline $\begin{array}{l}\text { Enfrentamento e psicopatologias em crianças } \\
\text { com malignidade e bronquiectasia }\end{array}$ & BIKMAZER A, et al. & 2020 & Pneumologia Pediátrica & Estudo quantitativo \\
\hline $\begin{array}{c}\text { Trajetórias de Criança e Cuidador } \\
\text { Adaptação psicológia em famílias de crianças } \\
\text { com Câncer }\end{array}$ & KATZ LF, et al. & 2018 & Health Pychology Journal & $\begin{array}{l}\text { Estudo prospectivo de curto } \\
\text { prazo }\end{array}$ \\
\hline $\begin{array}{c}\text { Impacto do Câncer na Infância em } \\
\text { Relacionamentos Românticos de Adultos } \\
\text { Emergentes Sobreviventes: Um estudo } \\
\text { qualitativo }\end{array}$ & THOMPSOM AL, et al. & 2013 & International Society for Sexual Medicine & Estudo quantitativo \\
\hline $\begin{array}{l}\text { Internalização de sintomas em adolescentes e } \\
\text { adultos jovens sobreviventes do câncer } \\
\text { pediátrico e comparações combinadas }\end{array}$ & D'SOUZA AM, et al. & 2019 & Psycho-Oncology & $\begin{array}{c}\text { Estudo de acompanhamento } \\
\text { transversal }\end{array}$ \\
\hline Atualizações em Psico-oncologia Pediátrica & MAVRIDES N, PAO M. & 2014 & International Review of Psychiatric & Revisão sistemática \\
\hline $\begin{array}{l}\text { Estresse Pós-Traumático como Contribuidor } \\
\text { para Efeitos de saúde comportamental e } \\
\text { utilização dos serviços de saúde em adultos } \\
\text { sobreviventes do cancer pediátrico: um } \\
\text { relatório do Estudo Do Sobrevivente do } \\
\text { Câncer Pediátrico }\end{array}$ & CROCHET E, et al. & 2019 & Journal of Cancer Survivorship & $\begin{array}{l}\text { Estudo de coorte } \\
\text { retrospectivo multi- } \\
\text { institucional }\end{array}$ \\
\hline $\begin{array}{c}\text { Sintomas Psicológicos, Efeitos Sociais, } \\
\text { Concretização Socioeconômica, e } \\
\text { Comportamentos de Saúde entre } \\
\text { Sobreviventes do Câncer Pediátrico: Estado } \\
\text { Atual da Literatura }\end{array}$ & BRINKMAN TM, et al. & 2018 & Journal of Clinical Oncology & Revisão de literatura \\
\hline $\begin{array}{l}\text { Aspectos de disfunção da saúde mental entre } \\
\text { sobreviventes do câncer pediátrico }\end{array}$ & FIDLER MM, et al. & 2015 & British Journal of Cancer & $\begin{array}{l}\text { Estudo de coorte } \\
\text { populacional }\end{array}$ \\
\hline $\begin{array}{l}\text { Condições e Sintomas de Saúde Mental em } \\
\text { Hospitalizações Pediátricas: Estudo de } \\
\text { Prevalência de único Centro }\end{array}$ & DOUPNIK SK, et al. & 2017 & Academic Pediatrics & $\begin{array}{l}\text { Estudo de prevalência de } \\
\text { único centro }\end{array}$ \\
\hline $\begin{array}{c}\text { Saúde mental de sobreviventes do câncer } \\
\text { infantil e do adulto jovem a longo prazo: } \\
\text { revisão sistemática }\end{array}$ & FRIEND AJ, et al. & 2018 & International Journal of Cancer & Revisão sistemática \\
\hline
\end{tabular}

Fonte: Bittencourt ALC, et al., 2020. 
Dos estudos selecionados, apenas 4 pesquisas retrataram o desenvolvimento de doenças psiquiátricas após o diagnóstico de câncer ainda na infância. Destas, 3 apresentam semelhança nos resultados obtidos. Entretanto, todas convergem para a melhora do quadro com o passar do tempo.

Além disso, dos artigos analisados no presente estudo, 6 abordavam sobre as psicopatologias presentes em sobreviventes do câncer infantil em sua idade adulta. Este tema se faz importante, uma vez que atualmente $80 \%$ das crianças com câncer sobreviverão pelo menos 5 anos (D'SOUZA AM, et al., 2019; FRIEND AJ, et al., 2018; THOMPSON AL, et al., 2013).

Deles, dois excluem os pacientes que sofreram de Câncer do Sistema Nervoso Central (SNC) e de Carcinoma Ósseo, por conta de possíveis resultados destoantes, pois alegam que as psicopatologias presentes em sobreviventes destes dois tipos de câncer estão relacionadas ao local do tumor e aos tratamentos utilizados, haja vista que deixam maiores sequelas neurocognitivas e físicas que os demais tipos de malignidade. Dessa forma, as psicopatologias nestes pacientes não seriam oriundas da experiência do câncer na infância. Nos outros quatro artigos, que não excluem estes tipos de câncer de sua amostra, apareceram grandes diferenças entre os grupos citados e os demais pacientes (D'SOUZA AM, et al., 2019; THOMPSON AL, et al., 2013).

De início, o hospital, para as crianças, apresenta uma realidade paradoxal: é um local de sofrimento, mas que também representa um ambiente de cura, onde receberão tratamentos e realizarão exames. $A$ hospitalização, ainda, é uma situação que gera grande ansiedade no paciente, pois, a depender do período da mesma, da gravidade da doença e dos procedimentos médicos, é reforçado o sentimento de medo dos procedimentos e até da morte (CICOGNA EC, et al., 2010).

Isso é demonstrado no estudo de prevalência de Doupnik SK, et al. (2017), realizado no Hospital Infantil da Filadélfia, com pacientes de 4 a 21 anos, que avaliou a proporção de crianças internadas com enfermidades físicas e problemas de saúde mental. Dentre os 119 participantes, 31 relataram problemas de saúde mental, sendo os transtornos de ansiedade e depressão os diagnósticos mais prevalentes. Dessa forma, estudo permitiu identificar que quase metade dessas crianças internadas possuíam algum tipo de problema de saúde mental.

Além disso, a internação e a própria doença afetam o biopsicossocial da criança. O estudo quantitativo de Bikmazer A, et al. (2019), demonstrou que o câncer infantil afeta o bem-estar psicossocial da criança e que impacto é quase que invariável, independente das apresentações clínicas e resultados específicos da doença. É importante ressaltar que as crianças que foram informadas sobre a doença e o possível plano de tratamento tiveram menos ansiedade e depressão, tendo o seu bem-estar emocional melhorado.

Todavia, a reação da criança ao diagnóstico de câncer pode ser variável de acordo com a sua idade, já que depende do seu grau de entendimento da situação e com os estímulos estressores para cada uma. Segundo Mavrides N e Pao M (2014), a quebra prolongada do vínculo com familiares é o estímulo estressor para crianças de 0 a 6 anos, já que são eles a sua principal interação, enquanto que a suspensão das atividades diárias está mais relacionada com crianças maiores de 6 anos. Soma-se a isso o fato de que esse estudo relatou que algumas crianças podem sofrer de ansiedade e depressão e, em menor quantidade, de delirium.

Katz LF, et al. (2018), traz em sua pesquisa dados mais controversos em relação aos artigos supracitados. Nesse estudo prospectivo, é descrita a trajetória psicológica da criança, do momento do diagnóstico até o primeiro ano de tratamento. Observou-se que a maioria das crianças com câncer mostraram um ajustamento psicológico relativamente bom, sugerindo que essas crianças podem não estar em risco elevado de dificuldades de saúde mental. E aquelas que apresentaram algum quadro, sendo mais crítico no primeiro ano após o diagnóstico, diminuíram os sintomas ao longo do tempo.

Os principais fatores de risco encontrados para desenvolvimento de psicopatologias em sobreviventes do câncer pediátrico foram: pessoas do sexo feminino, desempregados ou que nunca trabalharam, acima de 25 anos, divorciados ou solteiros, de baixa escolaridade, sobreviventes de tumores do SNC e sarcoma ósseo, 
portadores de deficiência física, brancas ou hispânicas, pessoas que foram tratadas com radioterapia craniana e antraciclinas. Brinkman TM, et al. (2018), traz, ainda, uma ressalva: alguns fatores como baixa renda e escolaridade, deficiências físicas e estado civil solteiro podem constituir tanto causas como consequências das psicopatologias (CROCHET E, et al., 2019; FIDLER MM, et al., 2015; FRIEND AJ, et al., 2018).

As principais causas apontadas para as diferenças de incidência de psicopatologias em sobreviventes de câncer do SNC e carcinomas ósseos foram: dores crônicas, condições ortopédicas, efeitos cognitivos tardios, dificuldades de mobilidade, dependência dos cuidadores primários e dificuldade de realizar tarefas, além do tratamento com radioterapia craniana. Brinkman TM, et al. (2018), afirma, ainda, que estas pessoas estão em maior risco para baixa escolaridade e desemprego (CROCHET E, et al., 2019; FRIEND AJ, et al., 2018).

As antraciclinas são citadas por Friend AJ, et al. (2018), como fator de risco devido ao seu fator cardiotóxico, e é devido ao seu uso que o pacientes que foram acometidos por sarcoma apresentaram a saúde mental mais prejudicada, entre os pacientes que não tinham tumores no SNC. Já a radioterapia craniana é citada em quatro dos artigos sobre sobreviventes do câncer pediátrico como fator de risco para desenvolvimento de psicopatologias (BRINKMAN TM, et al., 2018; CROCHET E, et al., 2019; D'SOUZA AM, et al., 2019).

Porém, dois estudos não encontraram grande relevância nessa modalidade de tratamento: D'Souza AM, et al. (2019), alega que a radioterapia craniana não tem mais sido utilizada com grande frequência devido aos seus efeitos neurocognitivos já conhecidos; e Fidler MM, et al. (2015), não encontrou variação significativa no índice de disfunções da saúde mental segundo a modalidade de tratamento utilizada, entre quimioterapia, radioterapia e intervenção cirúrgica. Crochet $\mathrm{E}$, et al. (2019), aponta que de 8 a $20 \%$ dos sobreviventes do câncer na infância desenvolvem Transtorno do Estresse Pós-Traumático (TEPT) na idade adulta, e boa parte destes pacientes com TEPT relataram tratamento com radioterapia craniana.

Dentre os artigos sobre sobreviventes do câncer pediátrico analisados, quatro apontam para as dificuldades de natureza psicossocial enfrentadas por estes, quais sejam: dificuldades com relacionamentos interpessoais, angústia somática ou somatização aumentadas, baixa autoestima, depressão e outros transtornos de humor, comportamento antissocial, ansiedade e outros transtornos neuróticos, TEPT, esquizofrenia e outros transtornos psicóticos, transtornos de comportamento, dificuldade de atingir objetivos, variações de humor, transtorno desafiador e de oposição, abuso de álcool e drogas, ideação suicida e necessidades emocionais não atendidas, competência social prejudicada, além de menor taxa de casamentos e coabitação. Essas dificuldades muitas vezes vêm da diferença de maturidade, perspectivas e valores em relação às outras pessoas de mesma faixa etária, e comumente perduram na vida adulta (BRINKMAN TM, et al., 2018; FIDLER MM, et al., 2015; FRIEND AJ, et al., 2018; THOMPSON AL, et al., 2013).

Segundo Thompson AL, et al. (2013), as dificuldades de socialização enfrentadas pelos sobreviventes do câncer pediátrico advêm do fato de que, quando crianças, perdiam grandes oportunidades de interagir com colegas da mesma idade pois perdiam aulas para ir a consultas médicas, por estarem hospitalizadas ou se recuperando de efeitos colaterais do tratamento, o que tem impacto no desenvolvimento de habilidades sociais.

A sobrevivência ao câncer durante a infância traz consequências positivas para o psicológico do paciente, em termos de amadurecimento, mudança de valores, posicionamento de prioridades e metas de vida. Ainda assim, essa diferença de experiências tende a colocar essa criança num nível de interação social diferente das demais de seu ciclo próximo, o que pode levar a problemas secundários de socialização e sensação de pertencimento ao meio que trarão maiores consequências na idade adulta (BRINKMAN TM et al, 2018; D'SOUZA AM, et al., 2019; THOPMSON AL, et al., 2013).

A idade avançada, acima dos 25 anos, também constitui um fator de risco para o desenvolvimento de psicopatologias nos sobreviventes do câncer pediátrico, e pode estar relacionada a efeitos tardios do tratamento. Além disso, a fase de adulto emergente passa a ser um período crítico, pois é quando as dificuldades de socialização na infância terão maior impacto na adaptação psicossocial a longo prazo. (BRINKMAN TM, et al., 2018; FIDLER MM, et al., 2015; THOMPSON AL, et al., 2013). 
Brinkman TM, et al. (2018), afirma que a dificuldade de desenvolver relacionamentos interpessoais e de se tornar independente de seus cuidadores primários reflete na menor taxa de casamentos e coabitação entre os sobreviventes do câncer pediátrico, e que isso representa um grande prejuízo no alcance de marcos psicossexuais. Este estudo analisa, ainda, que esse prejuízo se manifesta nas mulheres como baixa na função sexual, interesse, satisfação e atividade; e, nos homens, se manifesta como atividade sexual diminuída e maior risco de disfunção erétil. Isso também pode estar relacionado a efeitos neurotóxicos dos tratamentos utilizados.

O estudo de Fidler MM, et al. (2015), comprova o impacto destes marcos psicossexuais no estado mental das pessoas que sobreviveram ao câncer, já que constatou em seus estudos que sobreviventes que são casados possuíam muito menos indicadores de disfunção da saúde mental que os sobreviventes solteiros.

O sexo feminino constituiu um grupo de risco para psicopatologias em 3 dos estudos analisados sobre os sobreviventes do câncer pediátrico. Thompsom AL, et al. (2013), estudou exclusivamente os relacionamentos românticos de mulheres entre 18 e 25 anos que sobreviveram ao câncer pediátrico, e excluiu de sua amostra as mulheres que tiveram tumores no SNC. Segundo este estudo, muitas mulheres consideraram que a experiência do câncer trouxe pontos positivos em suas vidas, como maturidade, mudança de valores e novas perspectivas de vida, mas relatam que estes fatores também constituem desafios nos seus relacionamentos interpessoais, gerando barreiras na interação com pessoas de mesma faixa etária (BRINKMAN TM, et al., 2018; FIDLER MM, et al., 2015; FRIEND AJ, et al., 2018).

Além de dificuldades de socialização e desenvolvimento de relacionamentos interpessoais, o câncer pediátrico interfere no rendimento escolar da criança. O baixo desempenho escolar pode estar relacionado à resposta ao tratamento do câncer, efeitos tardios destes tratamentos ou mudanças nas relações com seus professores e colegas (BRINKMAN TM, et al., 2018).

De acordo com Fidler MM, et al. (2015), os sobreviventes do câncer na infância com melhor qualificação acadêmica ou que trabalhavam em cargos mais altos ou profissionais também apresentavam menos disfunção da saúde mental. Isto reforça a importância da realização acadêmica e do trabalho na saúde mental a longo prazo, o que sugere que se deve fornecer suporte educacional e aconselhamento profissional durante e após o tratamento, para que estes sobreviventes tenham as mesmas oportunidades de sucesso profissional e acadêmico que seus colegas.

Esse fato se reflete na idade adulta nas altas taxas de desemprego entre os sobreviventes do câncer pediátrico. Segundo Brinkman TM, et al. (2018), a taxa de desemprego entre as vítimas do câncer pediátrico é cerca de $50 \%$ maior que entre a população geral, o que pode variar dependendo da região analisada. Além disso, a renda global tende a ser menor.

Concomitantemente, três dos estudos sobre sobreviventes do câncer pediátrico abordam os comportamentos de saúde desses. Indica-se que os pacientes que sobrevivem ao câncer infantil possuem maior tendência a buscar os serviços de saúde mental, mesmo que alguns estudos apontem que estas pessoas não estão mais propensas a psicopatologias severas em relação a aquelas que nunca tiveram um câncer. De acordo com Brinkman TM, et al. (2018), os sobreviventes do câncer infantil são menos prováveis a usar drogas ilícitas como cocaína e crack, mas o uso de maconha, tabaco e álcool tem se mostrado alto em estudos. Além disso, 54\% dos sobreviventes excedem a ingestão de calorias diárias recomendada, e 52\% não praticam a atividade física recomendada (FRIEND AJ, et al., 2018).

Todos os artigos analisados retratam que embora as crianças sejam resilientes, uma parte das que sofrem de doenças como o câncer apresentam problemas de saúde mental. Sendo que os transtornos mais frequentes são o de ansiedade e a depressão. Isso ocorre, sobretudo, por conta da quebra do vínculo entre vida antes e durante o tratamento. Mas, para contrapor os problemas, foi evidenciado que um suporte psicológico e familiar, fotografias de parentes e tempo brincando com amigos são medidas positivas contra os problemas de saúde mental. Além disso, transmitir as crianças informações sobre sua doença e possíveis tratamentos mostrou promover maior bem-estar emocional. 


\section{CONSIDERAÇÕES FINAIS}

As mudanças comportamentais, físicas e psicológicas desencadeadas nas crianças que são diagnosticadas com câncer são bastante significativas, principalmente devido ao afastamento da sua rotina normal. O biopsicossocial da criança, que ainda se encontra em fase de adaptação, sofre muito com essa mudança brusca, sendo por isso os altos índices de transtornos de ansiedade e de depressão. Diante disso, a relevância do tema se baseia na importância de entender sobre as grandes consequências psicológicas nas crianças que foram diagnosticadas com alguma neoplasia. A literatura mostra algumas diferenças nos efeitos atrelados à idade em que a criança é diagnosticada e o tempo até sua cura, além de ser escassa quando se trata de estudos brasileiros. Isso mostra a importância em voltar a atenção para o tema com a elaboração de mais estudos para que se possam reduzir significativamente os efeitos desses transtornos na vida dessas crianças.

\section{REFERÊNCIAS}

1. ANDERS JC, SOUZA AIJ. Crianças e adolescentes sobreviventes ao câncer: desafios e possibilidades. Ciência, Cuidado e Saúde, 2009: 8 (1): 131-137.

2. BELL $\mathrm{H}$, et al. A systematic review of factors related to children's quality of life and mental health after brain tumor. Psycho-oncology, 2018; 27 (10): 2317-2326.

3. BIKMAZER A, et al. Coping and psychopathology in children with malignancy and bronchiectasis. Pediatric Pulmonology, 2020; 55 (1): 214-220.

4. BRINKMAN T, et al. Psychological symptoms, social outcomes, socioeconomic attainment, and health behaviors among survivors of childhood cancer: current state of the literature. Journal of Clinical Oncology, 2018; 36 (21): 2190.

5. CARDOSO FT. Câncer infantil: aspectos emocionais e atuação do psicólogo. Dissertação (Pós graduação em Psicologia Hospitalar) - Serviço de Psicologia Hospitalar da $28^{\circ}$ Enfermaria da Santa Casa da Misericórdia do Rio de Janeiro. Rio de Janeiro, 2007.

6. CASTRO EHB. A experiência do câncer infantil: repercussões familiares, pessoais e sociais. Revista Mal-estar e Subjetividade, 2010; 10 (3): 971-994.

7. CICOGNA EC, et al. Crianças e adolescentes com câncer: experiências com a quimioterapia. Revista LatinoAmericana de Enfermagem, 2010; 18 (5): 1-9.

8. CROCHET E, et al. Posttraumatic stress as a contributor to behavioral health outcomes and healthcare utilization in adult survivors of childhood cancer: a report from the Childhood Cancer Survivor Study. Journal of Cancer Survivorship, 2019; 13 (6): 981-992.

9. CUVIELLO A, et al. Initiating Palliative Care Referrals in Pediatric Oncology. Journal of Pain and Symptom Management, 2020.

10. DOUPNIK SK, et al. Mental health conditions and symptoms in pediatric hospitalizations: a single-center point prevalence study. Academic Pediatrics, 2017; 17 (2): 184-190.

11. D'SOUZA AM, et al. Internalizing symptoms in AYA survivors of childhood cancer and matched comparisons. Psychooncology, 2019; 28 (10): 2009-2016.

12. FIDLER MM, et al. Aspects of mental health dysfunction among survivors of childhood cancer. British journal of cancer, $2015 ; 113(7): 1121-1132$.

13. FRIEND AJ, et al. Mental health of long-term survivors of childhood and young adult cancer: A systematic review. International journal of cancer, 2018; 143 (6): 1279-1286.

14. HERNÁNDEZ S, et al. Indicadores de alteraciones emocionales y conductuales en menores oncológicos. Psicooncología: investigación y clínica biopsicosocial en oncología, 2009; 6 (2): 311-325.

15. KATZ LF, et al. Trajectories of child and caregiver psychological adjustment in families of children with cancer. Health Psychology, 2018; 37 (8): 725.

16. MAVRIDES N, PAO M. Updates in paediatric psycho-oncology. International review of psychiatry, $2014 ; 26$ (1): $63-73$.

17. OLIVEIRA J, et al. Transtornos depressivos em crianças com leucemia linfoide aguda e com insuficiência renal crônica terminal / estudo de séries de caso. Jornal de Psiquiatria. 2009; 58 (3): 212-216.

18. THOMPSON AL, et al. Impact of childhood cancer on emerging adult survivors' romantic relationships: a qualitative account. The Journal of Sexual Medicine, 2013; 10: 65-73.

19. WIENER L, et al. Pediatric psycho-oncology care: standards, guidelines, and consensus reports. Psycho-Oncology, 2015; 24 (2): 204-211.

20. WHO. WORLD HEALTH ORGANIZATION. Global Initiative for Childhood Cancer. United States, 2018. 